\title{
Clinical and Magnetic Resonance Imaging Factors Which May Predict the Need for Surgery in Lumbar Disc Herniation
}

\author{
Rouzbeh Motiei-Langroudi ${ }^{1}$, Homa Sadeghian ${ }^{2}$, Amir Saied Seddighi $^{1}$ \\ ${ }^{1}$ Functional Neurosurgery Research Center, Department of Neurosurgery, Shohada-e-Tajrish Hospital, Tajrish Square, Tehran, Iran \\ ${ }^{2}$ Shefa Neuroscience Research Center, Tehran, Iran
}

\begin{abstract}
Study Design: Case-control.
Purpose: Evaluate clinical and imaging factors which may predict the risk of failure of medical therapy in patients with lumbar disc herniation (LDH).

Overview of Literature: LDH is a common cause of low back pain and radicular leg pain, with a generally favorable natural course. At present, however, it is not possible to identify patients who may be candidates for surgery in an early stage of their disease by means of clinical signs or diagnostic imaging criteria.

Methods: We designed a study investigating patients with untreated low back pain to assess the predictive value of demographic, clinical or imaging findings in identifying patients who finally would meet the classic current criteria for surgery.

Results: Among 134 patients, 80.6\% were successfully treated with conservative therapy and $19.4 \%$ finally underwent surgery. Sex, occupation, involved root level, presence of Modic changes, osteophytes or annular tears were not significantly different between the 2 groups, while cerebrospinal fluid block, Pfirrmann's grade, location of herniation with regard to the midline, and type of herniation were significantly different. Anteroposterior fragment size was significantly higher and intervertebral foramen height and thecal sac diameters were significantly lower in the surgical group.

Conclusions: Although it is strongly recommended to practice conservative management at first for patients with LDH symptoms, the results of this study shows that higher Pfirrmann's grade, more laterally located discs, extrusion and protrusion herniation types, and larger fragments could predict the risk of conservative treatment failure. This way, unnecessarily prolonged conservative management (beyond 4-8 weeks) may be precluded.
\end{abstract}

Keywords: Intervertebral disc displacement; Magnetic resonance imaging; Conservative therapy; Surgery

\section{Introduction}

Lumbar disc herniation (LDH) is a common cause of low back pain (LBP) and radicular leg pain, which is reported to affect as many as $40 \%$ of the adult population at some point of life [1]. The natural course of LDH is generally favorable and the majority of patients recover spontaneously in about 4-6 weeks after onset with conservative therapy alone [2-5]. On the other hand, approximately $20 \%$ of the patients with radicular leg pain caused by

Received Apr 9, 2013; Revised May 25, 2013; Accepted Jun 2, 2013

Corresponding author: Amir Saied Seddighi

Functional Neurosurgery Research Center, Department of Neurosurgery, Zip Code: 1989934148,

Shohada-e-Tajrish Hospital, Tajrish Square, Tehran, Iran

Tel: +98-21-22718027, Fax: +98-21-22718027, E-mail: eddingtonsierra@hotmail.com 
LDH have strong indications for surgical intervention [1]. At present, however, it is not possible to identify those patients who may be candidates for surgery in an early stage of their disease using clinical signs or diagnostic imaging criteria [6].

At the onset of symptoms, a conservative multidisciplinary treatment approach consisting of medical and physical therapy should precede surgery [7]. Thereafter, the decision about the surgical or conservative management of herniated lumbar discs has to be based on the results of clinical and neurological examinations; current guidelines keep the results of imaging techniques such as magnetic resonance imaging (MRI) secondary [8]. The most common indication for surgical management of $\mathrm{LDH}$ is failure to improve with conservative management [9]. Most clinicians advocate waiting 4 to 8 weeks from the onset of symptoms before considering surgery. However, in cases with cauda equina syndrome, progressive motor deficit, or in patients whose pain remains intolerable despite adequate medical therapy, surgery is indicated before this time course has elapsed [10,11]. Many authors, however, have revealed a poor prognosis for patients operated on after prolonged conservative treatment $[12,13]$. Consequently, for obtaining better outcomes for $\mathrm{LDH}$, it would be helpful to predict the possibility that an individual patient may benefit fully from conservative management, or on the contrary, may be a candidate for surgery, at an early stage after the onset of symptoms.

MRI is frequently used in the diagnostic evaluation of LDH. Nonetheless, there is still a paucity of data regarding the prognostic value of MRI findings in $\mathrm{LDH}$ and its predictive value for patients who may need surgery for $\mathrm{LDH}$; therefore, it is not routinely used as a criterion for surgery. Komori et al. [12] suggested that the type of herniation (based on a 3 type classification presented by the authors) is correlated with outcome and is a major prognostic factor, while others have reported cases with lumbar disc extrusion who had fully recovered with medical therapy, both clinically and on imaging $[14,15]$. Others have suggested that discs with a high signal on T2 weighted images were more prone to regression in size [16]. However, these studies are few and the results are not conclusive.

To the best of our knowledge, no study has examined the relationship between clinical and lumbosacral MRI abnormalities and the likelihood of being deemed a surgical candidate among symptomatic patients with LDH.
Therefore, we designed a prospective study to investigate patients with untreated LBP with or without radicular leg pain to assess the predictive value of demographic, clinical or imaging findings in those who finally would meet the classic current criteria for surgery.

\section{Materials and Methods}

Patients were recruited during September 2011 to February 2012 at Shohada Tajrish Hospital, Tehran, Iran in a consecutive manner. All patients with a history of LBP or related radicular leg pain were initially included in the study. Patients who had indications for urgent surgery such as profound or progressive motor deficits or cauda equina syndrome at the first visit were excluded from the study. Included patients had not received any therapy, or had received non-standard treatments. Patients with major psychological problems (depression, psychosis, etc.) were excluded from the study. Upon arrival, a thorough medical history and physical examination were taken from the patients by a neurosurgeon. The demographic and symptom related data including age, sex, occupation, length of symptoms and their exacerbation, type of LBP (axial, radicular, etc.), lower extremity strength (scaled as standard by $0-5$ scores), and the involved dermatome were recorded in a computerized database. A lumbosacral MRI (Magnetom Avanto 1.5T, Siemens, Harvey, IL USA) without contrast was performed in all patients and its data were also recorded in the database. The MRI data included the level of the LDH (if present), presence or absence of osteophytes, annular tears, Schmorl's node, cerebrospinal fluid (CSF) block, and Modic type changes and their grade [17], Pfirrmann's grade [18], disc location in the axial plane (central, paracentral, foraminal, or extraforaminal), disc herniation type (bulging, protrusion, or extrusion), and the anteroposterior (AP) and mediolateral (ML) diameter of the thecal sac (both measured in axial MRI plane). Bulging was defined as the presence of disk tissue circumferentially $(50 \%-100 \%)$ beyond the edges of the ring apophyses. Protrusion was present if the greatest distance, in any plane, between the edges of the disk material beyond the disk space was less than the distance between the edges of the base in the same plane. Extrusion was present when any one distance between the edges of the disk material beyond the disk space was greater than the distance between the edges of the base or when no continuity existed between the herni- 
ated disk material and the disk space [17]. Intervertebral foraminal height was measured in sagittal MRI planes and was defined as the height of the peri-neural fat. In cases of disc protrusion and extrusion, some additional variables were also recorded: the presence of fragment migration (displacement of disk material away from the site of herniation [17]), and the AP and ML diameter of the herniated fragment (both measured in the axial MRI plane). Based on these measurements, 3 ratios were also calculated: 'AP fragment ratio, which was the AP herniated fragment diameter/AP thecal sac diameter, 'ML fragment ratio', which was the ML herniated fragment diameter/ML thecal sac diameter (Fig. 1A), and 'foramen ratio, which was the involved level intervertebral foramen height/normal foramen height (defined as the normal adjacent disc level) (Fig. 1B). Cases with diagnoses other than a single-level LDH were excluded from the study (multi-level LDHs, lumbar canal stenosis, spondylolysis, listhesis, etc.). Afterwards, all patients were asked to follow a comprehensive treatment regimen consisting of both physical therapy and medical therapy (including a combination of an nonsteroidal anti-inflammatory drug, tricyclic antidepressant, and a long-acting injectable corticosteroid). The patients received the treatment for at least 4 weeks. Cases with unresolved pain after a full course of conservative therapy were selected for surgery. In those with intolerable pain despite conservative medi- cal therapy, the decision for surgery was made sooner. To reduce selection bias, all measurements were performed by one neurosurgeon, and treatment decisions were made by another neurosurgeon blind to the database. Surgery was performed only in patients who gave their informed consent for the procedure. All surgeries were performed by one neurosurgeon (A.S.S., the senior neurosurgeon of the study).

Numeric data were analyzed with an independent sample $t$-test, and chi-square analysis was used for categorical data. A p-value less than 0.05 was considered as statistically significant. Analysis of the data was performed after termination of patient recruitment, with PASW Statistics ver. 18 (Predictive Analytics Software, SPSS Inc., Chicago, IL, USA).

The study design was approved by the Ethical Committee of Shahid Beheshti University of Medical Sciences and the study was performed with adherence to the statements of the Declaration of Helsinki.

\section{Results}

One hundred and thirty-four patients were recruited during the study period, based on the inclusion and exclusion criteria. The demographic and clinical data are presented in Table $1.89 \%$ of the patients presented with LBP, and $82 \%$ had radicular symptoms. Among those
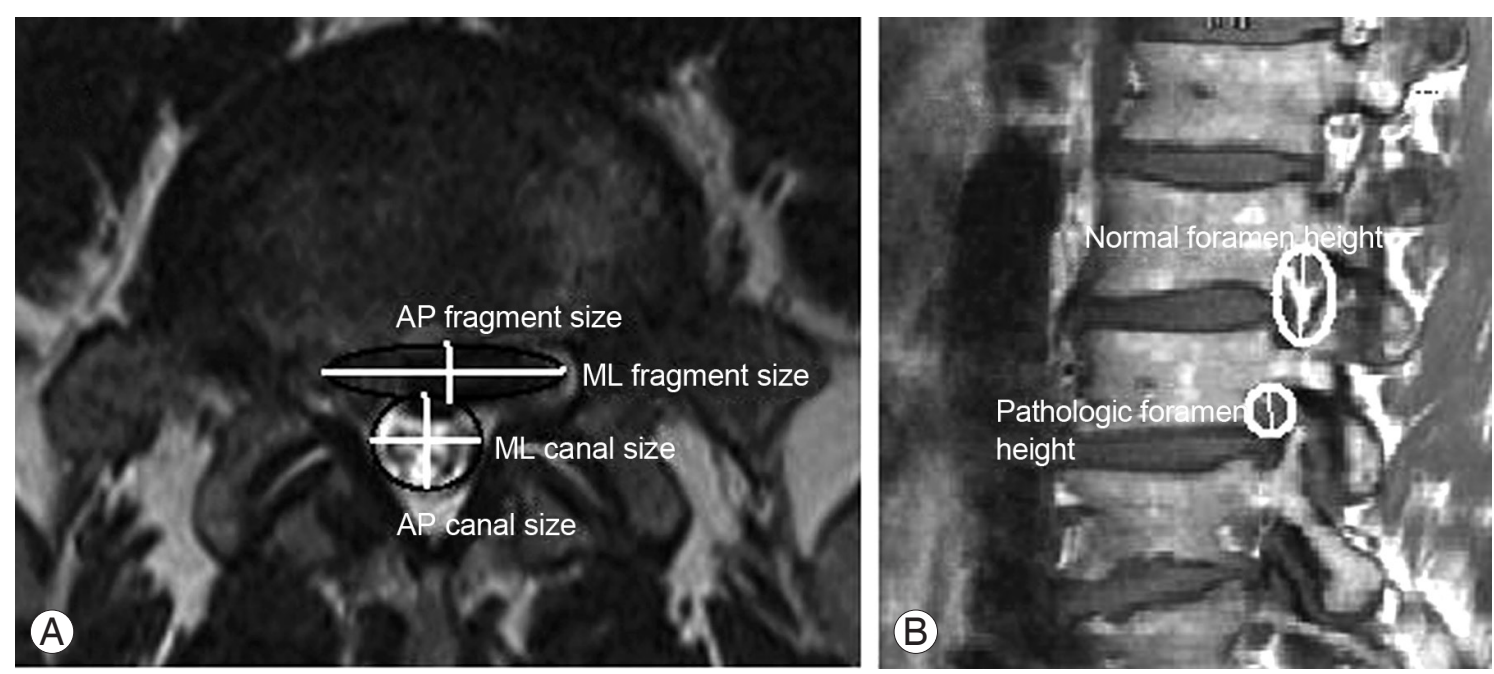

Fig. 1. (A) Axial magnetic resonance imaging in a patient with a protruded disc. The disc fragment and thecal sac are schematically shown as an oval and circle, respectively, and their diameters as AP and ML sizes. The ratios are calculated as: AP fragment ratio=AP fragment size/AP canal size; $\mathrm{ML}$ fragment ratio=ML fragment size/ML canal size. (B) A sagittal magnetic resonance imaging in a different patient. Neural foramens are schematically shown as ovals encircling peri-neural fat. Foramen ratio=pathologic foramen height/normal foramen height. AP, anteroposterior; ML, mediolateral. 
Table 1. Clinical data of the patients

$\begin{array}{lc}\text { Characteristic } & \\ \text { Age (yr) } & 43.0 \pm 13.4(15-81) \\ \text { Symptom length } & 23.5 \pm 43.0 \mathrm{mo}(1 \mathrm{wk}-20 \mathrm{yr}) \\ \text { Symptom exacerbation length } & 2.3 \pm 3.9 \mathrm{mo}(1 \mathrm{wk}-2 \mathrm{yr}) \\ \text { Male/female } & 71 / 63\end{array}$

Values are presented as mean \pm standard deviation (range), except for male/female ratio.

with radicular pain, the most prevalent dermatome was L5 (58.7\% of cases), followed by S1 (30.3\%); $60 \%$ had left sided, 33.8\% had right sided, and $6.2 \%$ had bilateral symptoms. On MRI, the most involved level was L4-L5 (57.8\% cases), followed by L5-S1 (35.3\%). Among those with LDH, 32.7\% had disc bulging, 54.8\% had protrusion, and $12.5 \%$ had disc extrusion. Among the 134 patients, $108(80.6 \%)$ were successfully treated with conservative therapy (we will refer to this group as the 'medical group' thereafter), and 26 (19.4\%) finally underwent surgery (hence they will be referred to as the 'surgical group'). The indication for surgery was failure to respond to conservative therapy or intolerable pain.

We further analyzed demographic and clinical findings between patients who needed surgery and those who received conservative care. Neither sex, occupation, or involved root was significantly different in the medical and surgical groups ( $p=0.53,0.50,0.43$, respectively). Mean age was 45.9 years in the surgical and 40.8 years in the medical group; however, this difference was not statistically significant $(p=0.076)$. The length of symp- toms was significantly longer in the surgical group (64.9 months vs. 25.6 months; $p=0.024)$. Among MRI findings, neither presence of Modic type changes nor their grade was significantly different between the 2 groups ( $p=0.576$ ). Also, presence of osteophytes and annular tears were not different between the 2 groups ( $p=0.366$ and 0.477 , respectively). Schmorl's nodes were more prevalent in the medical group ( $p=0.027$ ). CSF block was more prevalent in the surgical group $(73.9 \%$ of patients with CSF block underwent surgery, while this rate was $9.6 \%$ for those without block, $p<0.001)$. The surgical group had a higher Pfirrmann's grade than the medical group (surgical group: mean $=3.1$, mode $=3$, medical group: mean $=2.6$, mode $=2$; $p=0.008$ ). Analysis showed that the location of the herniation and its type were also significantly different between the 2 groups ( $p<0.001$ for both). $13.4 \%$ of patients with central herniation underwent surgery, while this rate was $52 \%, 75 \%$, and $100 \%$ for paracentral, foraminal, and extra-foraminal types, respectively (it should be noted that only 1 patient had an extra-foraminal herniation). Regarding herniation type, the rate of surgery was $3.3 \%$, $29.1 \%$, and $69.2 \%$ for disc bulging, protrusion, and extrusion, respectively. Rate of disc migration was also significantly different between the 2 groups, and it was higher in the surgical group $(p=0.05)$. Table 2 shows herniated disc fragment and thecal sac diameters and intervertebral foramen size in the 2 groups. As evident in the table, AP fragment size was significantly higher and intervertebral foraminal height, AP and ML thecal sac size were significantly lower in the surgical group. Mediolateral fragment size was not statistically different between the 2 groups. Moreover, AP and ML fragment ratios were significantly

Table 2. Herniated fragment and thecal sac diameters and intervertebral foramen height and their relevant ratios in the surgical and medical groups

\begin{tabular}{lccc} 
Characteristic & Medical group & Surgical group & $p$-value \\
\hline AP fragment size & 5.2 & 7.7 & 0.001 \\
\hline ML fragment size & 19.1 & 16.8 & 0.117 \\
\hline AP thecal sac size & 11.1 & 9.0 & 0.023 \\
ML Thecal sac size & 15.7 & 11.7 & 0.001 \\
\hline Intervertebral foramen height & 17.8 & 15.3 & 0.430 \\
\hline AP fragment ratio & 0.57 & 1.04 & 0.003 \\
\hline ML fragment ratio & 1.18 & 1.81 & 0.001 \\
\hline Foramen ratio & 0.87 & 0.70 & 0.001 \\
\hline
\end{tabular}

All sizes are represented in $\mathrm{mm}$.

$\mathrm{AP}$, anteroposterior; $\mathrm{ML}$, mediolateral. 
higher and foramen ratio was lower in the surgical group.

To confirm the previous analysis, we further analyzed the data with binary logistic regression analysis. The results showed that sex, Modic type changes, Pfirrmann's grade, presence of osteophytes, Schmorl's nodes, annular tears, migration type, fragment ML size and foramen height were not significant predictors, $(p=0.84,0.91,0.19$, $0.21,0.25,0.89,0.56,0.29,0.20$, respectively) while age, duration of symptoms, CSF block, disk location with regard to the midline, disc herniation type, fragment $\mathrm{AP}$ size, canal AP size, canal ML size, fragment AP ratio, fragment ML ratio, and foramen ratio were significant positive predictors $(p=0.022,0.01,<0.001,0.035,0.014$, $0.004,0.022,0.001,0.003,0.001,0.011$, respectively). As evident, the discrepancies are for age (which is a significant predictor in the new regression analysis) and Pfirrmann's grade, disc herniation migration, and foramen height (which were significant factors in the previous analysis, but not in the regression analysis). In fact, the regression analysis pushed age to statistical significance. The score for significance in positive predictors was 5.3, $6.6,18.8,4.5,6.1,8.1,8.9,10.4,5.2,10.3$, and 6.4 for age, symptom duration, CSF block, disc herniation location, disc herniation type, fragment AP size, fragment AP ratio, fragment ML ratio, canal AP size, canal ML ratio, and foramen ratio, respectively.

\section{Discussion}

$\mathrm{LDH}$ is a common disease causing low back and leg pain in a substantial proportion of patients. It is first treated by non-operative means including short periods of bed rest, medications, and physical therapy unless indications for urgent surgery (profound or progressive motor deficit, sphincter symptoms) exist from the onset; in these cases, surgery should be planned before completion of conservative management. The natural course of LDH is generally favorable and up to $80 \%$ of patients respond to conservative therapy in an average of 4 to 6 weeks [2-5]. The remaining $20 \%$ of patients have a strong indication for surgical intervention [1]. The most common indication for the surgical management of $\mathrm{LDH}$ is failure to improve with conservative management [9]. Nowadays, most surgeons only rely on clinical criteria to choose between conservative or surgical treatment options and the results of imaging modalities such as MRI are kept secondary [8]. Moreover, it is not possible at present to identify amongst the 2 groups, patients who will respond favorably to conservative management, or on the other hand, patients who are candidates for surgery, by means of clinical signs or diagnostic imaging criteria [6].

Although MRI is frequently used in the diagnostic evaluation of LDH, it is not still clear which MRI findings can help to predict the proportion of patients who may need surgery, and therefore, MRI findings are not routinely used as a criterion for surgery. For instance, Rapan et al. [14] and Neault [15] in 2 separate case reports reported cases of LDH with extruded and sequestered fragments which underwent conservative management, and showed that after the completion of treatment, the disc fragment regressed in size as confirmed by MRI, and this was also accompanied by a full recovery from the symptoms. They concluded that surgical treatment is not always the method of choice in these patients. However, the 2 studies report a total of 3 patients and both lack longer follow-ups. Saal and Saal [11] studied the natural history of lumbar disc extrusions in 11 patients who were treated non-operatively. They also observed a regress in size with a decrease in neural impingement in a substantial number of their patients.

In another study, Erly et al. [16] reviewed 36 patients with $44 \mathrm{LDHs}$ to assess whether or not MRI signal characteristics would predict subsequent disk regression. In their study, 57\% of herniated disks decreased in size and $39 \%$ remained unchanged. They observed that larger herniations and extrusions were more likely to regress than smaller ones, and also, disks that regressed were more likely to have a high signal on T2 weighted images than those that were stable [16].

Our study was performed in patients presenting with previously untreated LBP or radicular leg pain without indications for urgent surgery at their initial assessment. In all patients, a lumbosacral MRI was performed and conservative therapy was given. The patients were divided afterwards, based on their response to conservative therapy. The results of our study show that among clinical parameters, only duration of symptoms and perhaps age (as the second parameter that was close to statistical significance in the $t$-test analysis and significant in the regression analysis) were different between the 2 groups. Among MRI parameters, presence of CSF block, herniated disc location and type, and fragment size were the factors which were different between the 2 groups. Regarding the location, as the herniation occurred further from 
the midline and more proximal to the intervertebral foramen, the likelihood of need for surgery became higher; the highest likelihood was seen in extra-foraminal herniations, followed by foraminal and then paracentral. In fact, proximity of the herniated disc to nerve roots is a major predictor of failure to respond to medical therapy. This is further confirmed by our observation that intervertebral foramen height and ratio were lower in the surgical candidates. Moreover, extruded herniation types were more prevalent in those who underwent surgery, followed by protruded types. Regarding the size of the herniated disc, our results show that discs with larger anterior-posterior diameters and decreased thecal sac diameters (an indicator of compression of the thecal sac) were more prone to be candidates for surgery. To simplify this, one can predict that for patients in whom the AP diameters of the disc fragment and thecal sac are equal, the likelihood of the need for surgery is increased (based on the results shown in Table 2, which demonstrates an AP fragment ratio of approximately 1 in the surgical group). In fact, larger disc fragments with more pronounced compression of the thecal sac are another predictor of failure to respond to conservative management. One could also interpret higher Pfirrmann's grades (an indicator of disc degeneration grade) as a positive prognostic factor for the risk for surgery, as it showed statistical significance in the $t$-test analysis, while it was insignificant in the regression analysis. The regression analysis further showed that among these factors, CSF block had the highest prognostic value, with canal width, fragment AP size, symptom duration, foramen size, and disc herniation type following in order.

\section{Conclusions}

Even though it is strongly recommended to adhere to the current guidelines for the treatment of LDH (to practice conservative management protocols first for patients presenting with LDH related symptoms), the results of this study showed that some MRI parameters such as higher Pfirrmann's grades, more laterally located discs (or more proximal to nerve roots), extrusion and protrusion herniation types, and larger fragments (also indicated by the presence of CSF blocks) could predict the risk of conservative treatment failure and the need for surgery. These findings may be used in the future to avoid unnecessarily prolonged conservative management (beyond 4-8 weeks) which can worsen the outcome of surgery.

\section{Conflict of Interest}

No potential conflict of interest relevant to this article was reported.

\section{Acknowledgments}

The authors of this work would like to appreciate Ms. Rabiee for her considerable assistance. The authors also claim to have to financial conflict of interests in this study.

\section{References}

1. Frymoyer JW. Lumbar disk disease: epidemiology. Instr Course Lect 1992;41:217-23.

2. Fager CA. Observations on spontaneous recovery from intervertebral disc herniation. Surg Neurol 1994;42:282-6.

3. Weber H. Lumbar disc herniation. A controlled, prospective study with ten years of observation. Spine (Phila Pa 1976) 1983;8:131-40.

4. Weber H, Holme I, Amlie E. The natural course of acute sciatica with nerve root symptoms in a doubleblind placebo-controlled trial evaluating the effect of piroxicam. Spine (Phila Pa 1976) 1993;18:1433-8.

5. Hofstee DJ, Gijtenbeek JM, Hoogland PH, et al. Westeinde sciatica trial: randomized controlled study of bed rest and physiotherapy for acute sciatica. J Neurosurg 2002;96:45-9.

6. Peul WC, van Houwelingen HC, van der Hout WB, et al. Prolonged conservative treatment or 'early' surgery in sciatica caused by a lumbar disc herniation: rationale and design of a randomized trial [ISRCT 26872154]. BMC Musculoskelet Disord 2005;6:8.

7. Porchet F. Role of surgical treatment of low back pain and lumbo-sciatica. Praxis (Bern 1994) 2001;90:187882.

8. Kramer J, Ludwig J. Surgical treatment of lumbar intervertebral disk displacement. Indications and methods. Orthopade 1999;28:579-84.

9. Storm PB, Chou D, Tamargo RJ. Surgical management of cervical and lumbosacral radiculopathies: indications and outcomes. Phys Med Rehabil Clin N Am 2002;13:735-59. 
10. Weber H. The effect of delayed disc surgery on muscular paresis. Acta Orthop Scand 1975;46:631-42.

11. Saal JA, Saal JS. Nonoperative treatment of herniated lumbar intervertebral disc with radiculopathy. An outcome study. Spine (Phila Pa 1976) 1989;14:431-7.

12. Komori H, Okawa A, Haro H, Shinomiya Ki K. Factors predicting the prognosis of lumbar radiculopathy due to disc herniation. J Orthop Sci 2002;7:56-61.

13. Davis RA. A long-term outcome analysis of 984 surgically treated herniated lumbar discs. J Neurosurg 1994;80:415-21.

14. Rapan S, Gulan G, Lovric I, Jovanovic S. Spontaneous regression of intervertebral disc herniation: case reports. Coll Antropol 2011;35:211-5.
15. Neault CC. Conservative management of an L4-L5 left nuclear disk prolapse with a sequestrated segment. J Manipulative Physiol Ther 1992;15:318-22.

16. Erly WK, Munoz D, Beaton R. Can MRI signal characteristics of lumbar disk herniations predict disk regression? J Comput Assist Tomogr 2006;30:486-9.

17. Krishnaney A, Modic MT. Radiology of the spine. In: Youmans JR, Winn HR, editors. Youmans neurological surgery. 6th ed. Philadelphia: Elsevier Saunders; 2011. p.311-54.

18. Pfirrmann CW, Metzdorf A, Zanetti M, Hodler J, Boos N. Magnetic resonance classification of lumbar intervertebral disc degeneration. Spine (Phila $\mathrm{Pa}$ 1976) 2001;26:1873-8. 